\title{
Preparation and Properties of PTFE-PMMA Core-Shell Nanoparticles and Nanocomposites
}

\author{
Diego Antonioli, ${ }^{1}$ Michele Laus, ${ }^{1}$ Giampaolo Zuccheri, ${ }^{2}$ Valerj Kapeliouchko, ${ }^{3}$ \\ Maria Cristina Righetti, ${ }^{4}$ Luca Boarino, ${ }^{5}$ and Katia Sparnacci ${ }^{1}$
}

\author{
${ }^{1}$ Dipartimento di Scienze e Innovazione Tecnologica, Università del Piemonte Orientale "Amedeo Avogadro," \\ Interuniversity Consortium for Materials Science Technology (INSTM), UdR Alessandria, Viale T. Michel 11, \\ 15100 Alessandria, Italy \\ ${ }^{2}$ Dipartimento di Biochimica “G. Moruzzi," Modena-S3, Istituto di Nanoscienze del Consiglio Nazionale delle Ricerche, \\ Università di Bologna, Italian Interuniversity Consortium for Materials Science and Technology (INSTM), UdR Alessandria, \\ Via Irnerio 48, 40126 Bologna, Italy \\ ${ }^{3}$ Solvay Solexis SpA, Piazzale Donegani 5/6, Alessandria 15047 Spinetta Marengo, Italy \\ ${ }^{4}$ Institute for the Chemical and Physical Processes, National Research Council, Via G. Moruzzi 1, 56124 Pisa, Italy \\ ${ }^{5}$ NanoFacility Piemonte, Electromagnetism Division, Istituto Nazionale di Ricerca Metrologica, Strada delle Cacce 91, \\ 10135 Torino, Italy
}

Correspondence should be addressed to Michele Laus, laus@mfn.unipmn.it

Received 4 April 2012; Accepted 16 July 2012

Academic Editor: Baoquan Sun

Copyright ( 2012 Diego Antonioli et al. This is an open access article distributed under the Creative Commons Attribution License, which permits unrestricted use, distribution, and reproduction in any medium, provided the original work is properly cited.

The preparation of polytetrafluoroethylene-poly(methyl methacrylate) (PTFE-PMMA) core-shell particles was described, featuring controlled size and narrow size distribution over a wide compositional range, through a seeded emulsion polymerization starting from a PTFE seed of 26 nanometers. Over the entire MMA/PTFE range, the particle size increases as the MMA/PTFE ratio increases. A very precise control over the particle size can be exerted by properly adjusting the ratio between the monomer and the PTFE seed. Particles in the 80-240 nm range can be prepared with uniformity indexes suited to build 2D and 3D colloidal crystals. These core-shell particles were employed to prepare nanocomposites with different compositions, through an annealing procedure at a temperature higher than the glass transition temperature of the shell forming polymer. A perfect dispersion of the PTFE particles within the PMMA matrix was obtained and optically transparent nanocomposites were prepared containing a very high PTFE amount.

\section{Introduction}

Fillers are well recognized to be integral components for enhancement of polymer properties in many applications. The oldest and most conventional processing route is melt compounding which is based on a direct dispersion of the filler into the polymer during the melt leading to composites. This processing route is very well adapted for producing high-performance parts by injection molding or extrusion. In all cases, an adequate surface preparation of the filler is crucial to favor their dispersion inside the polymer.

Nanocomposites represent the evolution of the conventional composites in that the filler size is in the nanoscale region, for example, nanoclay [1] and carbon nanotubes [2], and nanoparticles [3]. Due to the extremely high surface to volume ratio of the nanofillers, nanocomposites have pronounced properties that are not realized with traditional microscale fillers. The mechanical properties, electrical and thermal conductivity, and flammability resistance all differ in nanocomposites in comparison to conventional composites [4]. In turn, the processing aspects for this novel class of materials are definitely more critical with respect to microscale composites because of the inherently higher aggregation propensity of the nanofillers.

Although top-down approaches involving melt processing techniques are often employed because these are 


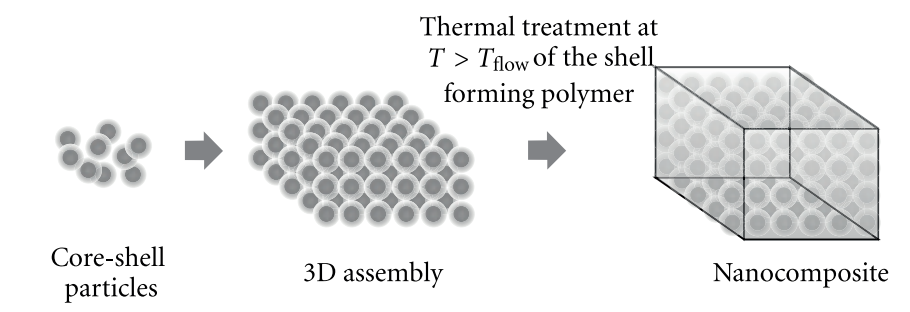

Scheme 1: Porous and compact nanocomposites from core-shell particles.

generally considered more economical, more flexible for formulation, and involve compounding and fabrication facilities commonly used in commercial practice; the ideal morphology, consisting of nanofillers individually dispersed in the polymer matrix, is frequently not achieved and varying degrees of dispersion are more common. This originates an inherent irreproducibility in the nanocomposite properties. In addition, the presence of nanofiller aggregates results particularly critical in applications in which transparency is required.

An alternative very efficient method to prepare nanocomposites featuring homogeneous nanofiller dispersion consists in the use of tailored polymer particles [5]. This bottom-up strategy involves either blends of colloidal polymer particles with a second type of particles (inorganic or polymeric) or hybrid particles consisting of a polymer phase and a second phase. A key advantage of this colloidal approach is that it offers control of structure at the nanoscale (within particles) and at the meso- and even macroscale through the formation of ordered assemblies of particles that should be considered as "building blocks" of larger structures.

In this general frame, core-shell particles are particularly suited system to produce a variety of nanostructured materials. Core-shell particles featuring a narrow size distribution can self-assemble to generate highly ordered structures, which represent promising candidates in applications such as catalysis, sensing, optics, molecular separation, single-molecule detection, and optoelectronics [6-8]. Twodimensional structuration leads to 2D colloidal crystals, which could be employed in nanosphere lithography (NSL) [9], among others. 3D structuration leads to opals in which photonic properties can be originated from the periodic modulation in structure and composition [10]. In addition, aside from the above porous materials, compact nanocomposites can be obtained [7] by annealing a 3D assembly of core-shell particles at a temperature lower than the glass transition or melting of the core but higher than the glass transition of the shell forming polymer to assure that the flow of the shell-forming polymer fills the voids (Scheme 1).

This thermal treatment leads to the formation of a continuous polymer matrix in which the cores could be arranged in regular registry within the matrix. Since the characteristic size of the different domains is on the nanometer range, much smaller than the wavelength of light, the material should be optically transparent.
In recent studies, polytetrafluoroethylene (PTFE) latexes with particles in the submicrometer size range were successfully employed as seeds in the emulsifier-free emulsion polymerization of various monomers including styrene [11], acrylic mixtures [12], or methyl methacrylate [13] thus leading to a wide variety of core-shell nanoparticle architectures featuring a relatively narrow size distribution. Two dimensional structuration of these particles leads to 2D colloidal crystals which were employed in nanosphere lithography [14] whereas 3D structuration allowed opals to be obtained featuring interesting photonic properties [15].

Following our continuing interest in the preparation and study of PTFE-based nanocomposites from core-shell nanoparticles, in this paper we further explore this strategy extending the previous studies through the preparation of polytetrafluoroethylene-poly(methyl methacrylate) (PTFEPMMA) core-shell particles over a wide compositional range, starting from a PTFE seed of 26 nanometers. The control of the size and size distribution of the resulting core-shell nanoparticles will be described and a variety of nanocomposites are prepared by thermally treating the corresponding core-shell nanoparticle assemblies.

\section{Experimental}

2.1. Materials. PTFE latex BP44 ( $350.4 \mathrm{~g} / \mathrm{L})$ was provided by Solvay Solexis and consists of particles with spherical shape, average diameter of $26 \mathrm{~nm}$, and narrow size distribution. Their general preparation was previously reported [11]. Methyl methacrylate (MMA) (99\%, Fluka) was distilled under reduced pressure in nitrogen atmosphere and stored at $-18^{\circ} \mathrm{C}$ until use. Potassium persulfate (98\%, Carlo Erba) was used without further purification.

\subsection{Preparation of PTFE-PMMA Core-Shell Nanoparticles.} The PTFE-PMMA core-shell colloids were synthesized in a $1 \mathrm{~L}$ five-neck-jacketed reactor equipped with a condenser, a mechanical stirrer, a thermometer, and inlets for nitrogen and monomer. First, the appropriate amount of PTFE latex was introduced into the reactor containing $500 \mathrm{~mL}$ of deionized water at room temperature with a stirring rate of $300 \mathrm{rpm}$. The mixture was purged with nitrogen for $20 \mathrm{~min}$ and nitrogen was flushed during the entire polymerization procedure. Next, the mixture was heated to $75^{\circ} \mathrm{C}$ and MMA $(50.0 \mathrm{~mL}, 0.467 \mathrm{~mol})$ was added. Then, after additional $15 \mathrm{~min}$ of equilibration time, the potassium persulfate aqueous solution $(10 \mathrm{~mL}, 0.59 \mathrm{mmol})$ was added 
TABLE 1: Synthesis details and yield of the various samples.

\begin{tabular}{lccccc}
\hline Latex & Sample & Volume of $\mathrm{H}_{2} \mathrm{O}(\mathrm{mL})$ & Volume of MMA $(\mathrm{mL})$ & Weight of PTFE $(\mathrm{g})$ & Yield $(\%)$ \\
\hline \multirow{5}{*}{ PMMA } & 500.0 & 50.0 & 0 & 88.7 \\
& BPM1 & 500.0 & 50.0 & 0.12 & 86.2 \\
BPM2 & 500.0 & 50.0 & 0.24 & 91.6 \\
BPM3 & 500.0 & 50.0 & 0.71 & 88.7 \\
& BPM4 & 500.0 & 50.0 & 1.45 & 93.5 \\
BPM5 & 500.0 & 50.0 & 2.99 & 93.7 \\
BP44 & BPM6 & 500.0 & 50.0 & 6.38 & 85.8 \\
& BPM7 & 500.0 & 50.0 & 10.27 & 88.2 \\
& BPM8 & 500.0 & 50.0 & 20.06 & 90.5 \\
& BPM9 & 500.0 & 50.0 & 31.20 & 91.0 \\
& BPM10 & 500.0 & 50.0 & 46.80 & 89.6 \\
& BPM11 & 500.0 & 50.0 & 70.20 & 79.9 \\
& BPM12 & 500.0 & 50.0 & 109.20 & 85.9 \\
BPM13 & 500.0 & 23.4 & 87.61 & 86.1 \\
& BPM14 & 500.0 & 15.6 & 82.74 & 87.3 \\
BPM15 & 500.0 & 11.7 & 98.56 & 85.7 \\
\hline
\end{tabular}

and the mixture was reacted for $24 \mathrm{~h}$. The obtained latex was purified from the unreacted monomer by repeated dialyses. All the PTFE-PMMA latexes were obtained following the above general procedure by varying the initial PTFE latex amount.

Table 1 collects the details of the various preparations, including yield.

2.3. Characterization. The concentration of latex dispersion was determined gravimetrically. The particle size and size distribution of the core-shell particles were determined by scanning electron microscope (SEM), scanning force microscope (SFM), and dynamic light scattering (PCS). The microscope was an Inspect F SEM-FEG (Field Emission Gun) from FEI Company, with a beam diameter of $3 \mathrm{~nm}$. The SEM micrographs were elaborated by the Scion Image processing program. From 300 to 350, individual microsphere diameters were measured for each sample. SFM analysis was performed with Tapping-Mode Scanning Force Microscopy (TM-SFM) in a NanoScope IIIa Scanning Force Microscope equipped with a Multimode head (Digital Instruments/Bruker, S. Barbara, CA, USA). TEM was performed by use of an Hitachi H 800 Microscope equipped with cold stage. For TEM observation, a certain quantity of dispersion was diluted properly by distilled water and then stained with $\mathrm{pH} 2.0$ phosphotungstic acid (PTA) solution. The mixture was allowed to stand for a while, then a drop of the stained latex was placed on a carbonformvar-coated, copper grid and dried at room temperature. Dynamic light scattering analysis was performed at $25^{\circ} \mathrm{C}$, with a Malvern Zetasizer 3000 HS at a fixed scattering angle of $90^{\circ}$, using a $10 \mathrm{mV} \mathrm{He}-\mathrm{Ne}$ laser and PCS software for Windows (version 1.34, Malvern, UK). Each value is the average of five measurements. Electrophoretic mobility was measured with a Malvern Zetasizer 3000 HS. Each value is the average of five measurements. The instrument was checked using a latex with known Zeta potential. Thermogravimetric analysis (TGA) was performed with a MettlerToledo TGA/SDTA851e at a scanning rate of $10^{\circ} \mathrm{C} / \mathrm{min}$ from room temperature up to $1100^{\circ} \mathrm{C}$ under nitrogen flow. Differential scanning calorimetry (DSC) was carried out using a Mettler-Toledo DSC 821 apparatus. Samples of about $5 \mathrm{mg}$ were employed. The instrument was calibrated with high-purity standards (indium, naphthalene, benzoic acid, and cyclohexane) at $10^{\circ} \mathrm{C} / \mathrm{min}$. Dry nitrogen was used as purge gas. The samples for the dynamic mechanical analysis were prepared introducing the powder sample into a rectangular mould. The entire assembly was then placed between press plates with a nominal pressure of $4.9 \times 10^{7} \mathrm{~Pa}$ and allowed to stand at room temperature for $20 \mathrm{~min}$. The temperature was then raised to $160^{\circ} \mathrm{C}$ and the pressure released to $4.9 \times 10^{6} \mathrm{~Pa}$. After $15 \mathrm{~min}$, the sample was cooled to room temperature and recovered as rectangular $30 \times 5 \times$ $2 \mathrm{~mm}$ sheets. The modulus was measured with a dynamic mechanical analyzer Rheometric DMTA V, employing the three point bending geometry. A static to dynamic stress ratio of $120 \%$ and a scanning rate of $4^{\circ} \mathrm{C} / \mathrm{min}$ were chosen. The strain was sufficiently small to be within linear viscoelastic range.

\section{Results and Discussion}

BP44 latex consists of spherical particles with average diameters of $26 \mathrm{~nm}$ and, as received, contains residual PFPE surfactant. As the presence of the residual surfactant could interfere with the emulsifier-free-seeded emulsion polymerization, the PTFE latex was thoroughly dialyzed. The conductivity of the BP44 latex was $830 \mu \mathrm{S} / \mathrm{cm}$ before and $120 \mu \mathrm{S} / \mathrm{cm}$ after the dialysis. The emulsifier-free seeded emulsion polymerizations were performed, as previously 


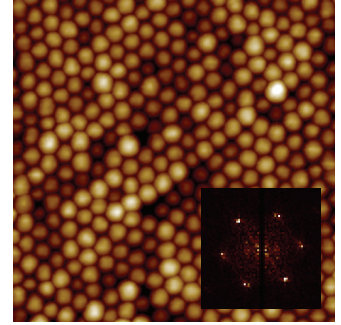

(a)

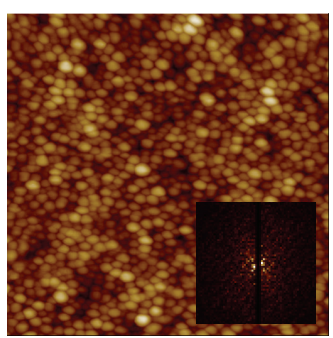

(b)

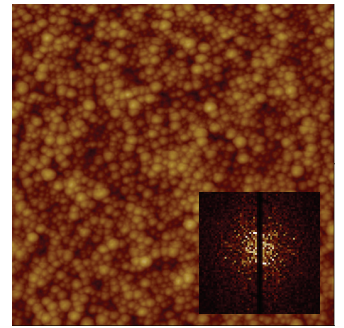

(c)

$0 \mathrm{~nm}$ $50 \mathrm{~nm}$

FIGURE 1: Tapping-mode AFM images of the top layer of a film obtained by slow solvent evaporation on freshly cleaved mica. (a) Image of the BPM4 specimen showing extreme regularity and very low dimension dispersion. In the inset, the 2D FFT showing distinct frequency peaks witnessing the high regularity of the hexagonal close-packed layer (FFT maxima measure an approximately $100 \mathrm{~nm}$ distance between particles). The direct-space measurement of the closest interparticle distances on a set of particles shows a (108 \pm 8$) \mathrm{nm}$ distance (average \pm standard deviation); (b) same as above on the BPM6 specimen, showing a markedly lower order in the layer (the 2D FFT, in the inset, does not show any distinct maxima). Direct-space measurements of closest interparticle distances report a (46 \pm 25$)$ nm distance, also witnessing a higher dimensional dispersion on the particle dimensions and packing; (c) same as above for the BPM10 specimen. Closest interparticle distances $(54 \pm 25) \mathrm{nm}$ with a low order multilayer (2D FFT in the inset with no distinct maxima). The size and relative heights of the micrographs are displayed according to the attached size bar and colormap.

described [11-13], by adding appropriate amounts of the PTFE latex (Table 1) and MMA into deionized water and running the reactions at $75^{\circ} \mathrm{C}$ for $24 \mathrm{hrs}$ using potassium persulfate as the free radical source. At the end of the reaction, the latexes were purified by repeated dialyses. In all the polymerization reactions, MMA and potassium persulfate as well as the water content were kept constant, whereas variations were allowed in the amount of PTFE. Under these conditions, the initial PTFE weight percent with respect to the total weight of MMA and PTFE was varied from 0.25 to $90 \%$. Accordingly, the sample series marked BPMn is obtained in which $\mathrm{n}$ is a number related to the initial PTFE weight percent. Stable latexes were obtained with nanosphere yields ranging from 85 to $93 \%$ (after the workup) and nearly quantitative MMA conversions (Table 1).

As a typical example, Figure 1 reports the tapping-mode AFM images of multilayers obtained by slow solvent evaporation on freshly cleaved mica of samples BPM4, BPM6, and BPM10, whereas Figure 2 reports representative PCS curves of core-shell samples and, for comparison purposes, of the BP44 seed latex.

No residual PTFE, deriving from BP44 latex, is present, and all the PCS curves are quite narrow. This suggests that the methyl methacrylate polymerization occurs quantitatively onto the PTFE seeds, as previously described for different systems [13]. The Zeta potential of the various samples was also measured and resulted comprised between -45 and $-60 \mathrm{mV}$ in agreement with an electrostatic stabilization driven by the repulsions of negative charges deriving from the ionic initiator fragments. The core shell nature of the samples was confirmed by direct TEM observation. Figure 3 reports the TEM image of sample BPM10 after treatment with phosphotungstic acid at a magnification of 180.000 . Although the particles appear deformed under the electronic beam, the core and shell structure can be clearly seen.

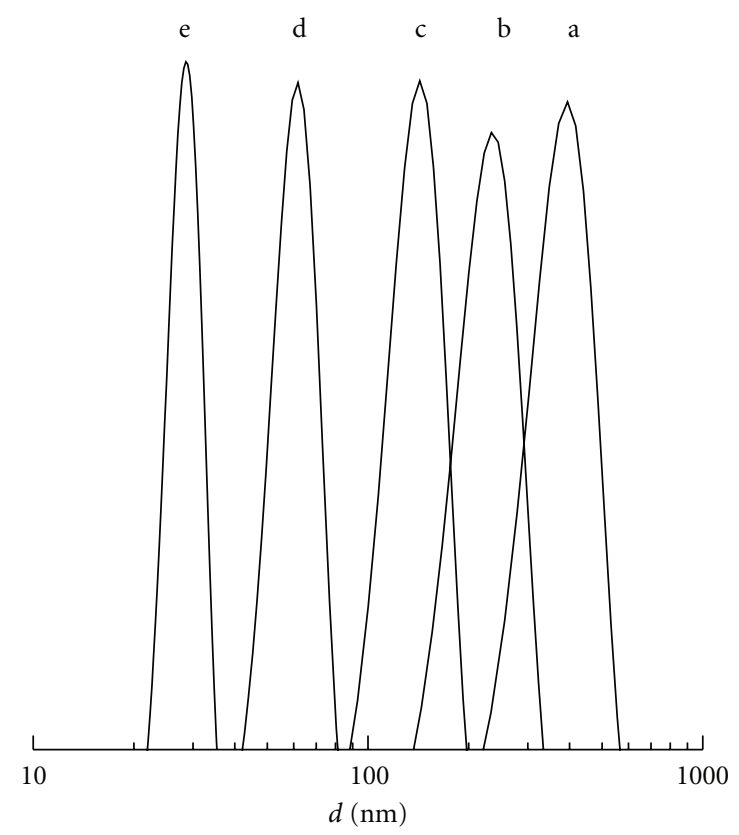

FIGURE 2: PCS spectrum of samples PMMA (a), BPM1 (b), BPM3 (c), BPM7 (d), and BP44 latex (e).

The composition of the various samples was determined by TGA and DSC data. Figure 4 reports the TGA curves of BPMn series including the BP44 latex and the TGA curve of a purely PMMA sample. The composition can be estimated from the TGA curves considering that the weight losses at 360 and $500^{\circ} \mathrm{C}$ correspond to PMMA and PTFE decomposition, respectively. On the other hand, in the DSC traces (Figure 5), the endothermic peak at about $325^{\circ} \mathrm{C}$ corresponds to PTFE melting. Consequently, the amount of PTFE in the core-shell 
TABLE 2: Size and composition data of the various samples.

\begin{tabular}{|c|c|c|c|c|c|c|}
\hline Sample & \% PTFE estimated (W/W) & $\% \mathrm{PTFE}^{(\mathrm{a})}(\mathrm{DSC})$ & $\% \mathrm{PTFE}^{(\mathrm{b})}(\mathrm{TGA})$ & $d$ estimated $(\mathrm{nm})$ & $d \operatorname{PCS}(\mathrm{nm})$ & $T_{g} \operatorname{DSC}^{(\mathrm{c})}\left({ }^{\circ} \mathrm{C}\right)$ \\
\hline PMMA & 0.0 & 0.0 & 0.0 & - & 391.3 & 121 \\
\hline BPM1 & 0.25 & 0.3 & 0.4 & 240 & 236.6 & 121 \\
\hline BPM2 & 0.5 & 0.4 & 0.4 & 191 & 194.4 & 121 \\
\hline BPM3 & 1.5 & 1.4 & 1.2 & 128 & 136.8 & 121 \\
\hline BPM4 & 3.0 & 3.1 & 3.4 & 101 & 112.0 & 121 \\
\hline BPM5 & 6.0 & 5.7 & 5.9 & 80 & 82.5 & 125 \\
\hline BPM6 & 12.0 & 12.0 & 15.4 & 63 & 68.5 & 134 \\
\hline BPM7 & 18.0 & 20.4 & 24.1 & 55 & 53.8 & 134 \\
\hline BPM8 & 30.0 & 22.8 & 28.8 & 45 & 48.0 & 131 \\
\hline BPM9 & 40.0 & 46.5 & 43.7 & 40 & 41.1 & 130 \\
\hline BPM10 & 50.0 & 53.0 & 48.1 & 37 & 38.4 & 128 \\
\hline BPM11 & 60.0 & 66.8 & 61.9 & 34 & 35.4 & 130 \\
\hline BPM12 & 70.0 & 70.3 & 67.2 & 31 & 31.8 & $\mathrm{Nd}$ \\
\hline BPM13 & 80.0 & 80.9 & 75.6 & 29 & 30.1 & $\mathrm{Nd}$ \\
\hline BPM14 & 85.0 & 84.5 & 86.5 & 29 & 29.4 & $\mathrm{Nd}$ \\
\hline BPM15 & 90.0 & 87.4 & 87.9 & 28 & 29.2 & $\mathrm{Nd}$ \\
\hline
\end{tabular}

(a) The PTFE percentage in the core-shell samples was estimated from the amount of feed PTFE and MMA assuming quantitative MMA conversion, absence of residual PTFE seeds, and no PMMA particle formation.

${ }^{(b)}$ The amount of PTFE was estimated from the melting enthalpy of the PTFE component (endothermic peak at $325^{\circ} \mathrm{C}$ ) considering that the melting enthalpy of the BP44 sample is $46.05 \mathrm{~J} \mathrm{~g}^{-1}$ (first DSC heating curve).

(c) First DSC heating curve at $20^{\circ} / \mathrm{min}$.

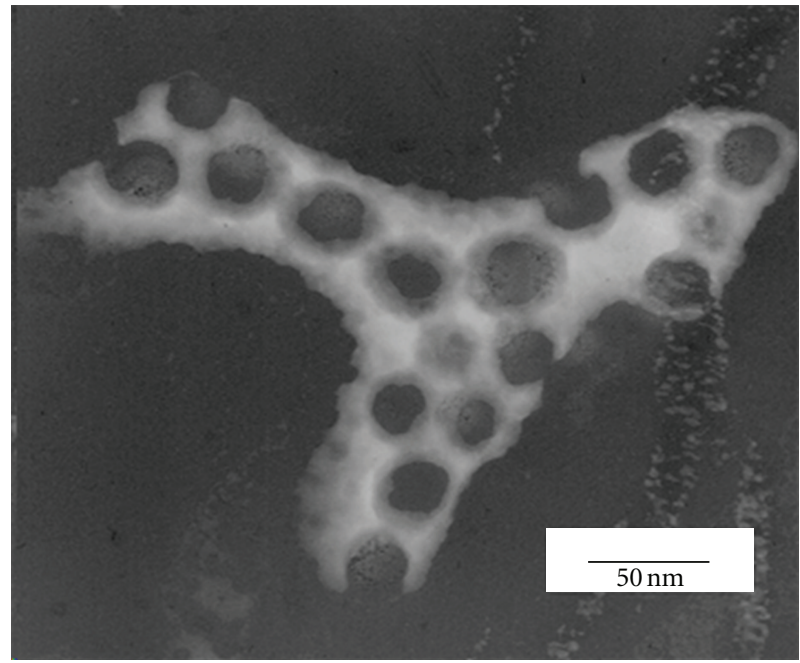

FIGURE 3: TEM micrographs of sample BPM10.

samples can be evaluated from the corresponding melting enthalpy in comparison with the melting enthalpy of the pure PTFE seed (Table 2) and, by difference, the amount of PMMA can also be determined assuming that there is no interaction between the PTFE core and the PMMA shell.

Provided that no pure PMMA or PTFE nanoparticles are present at the end of the reaction, and taking into account the yield values, the composition of the core-shell nanoparticles can be calculated from the amount of the initially added PTFE and MMA and are collected in Table 2.

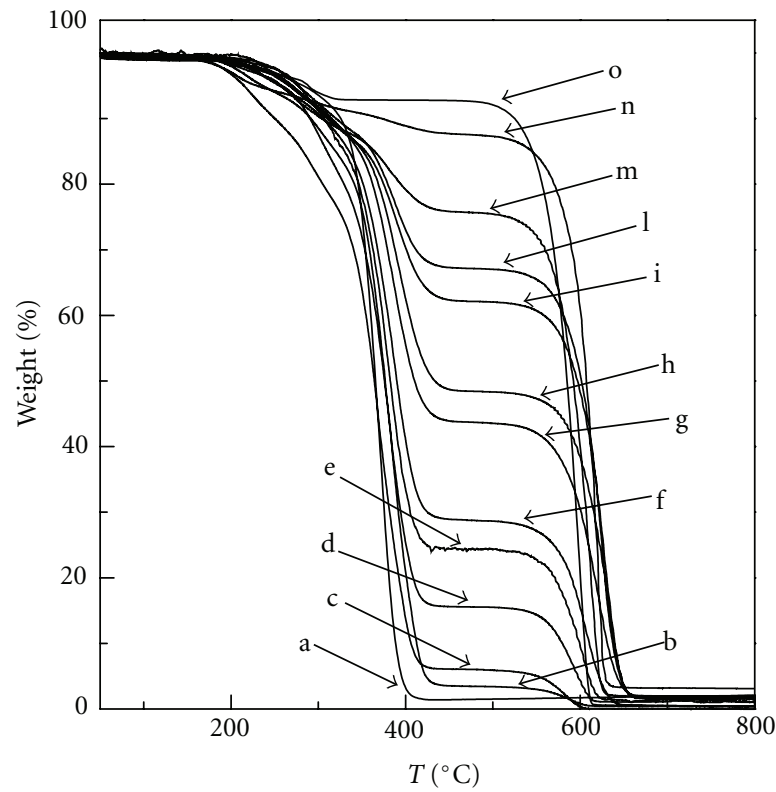

FIgURE 4: TGA curves at $10^{\circ} \mathrm{C} / \mathrm{min}$ heating rate for various samples in nitrogen atmosphere: PMMA (a); BPM4 (b); BPM5 (c); BPM6 (d); BPM7 (e); BPM8 (f); BPM9 (g); BPM10 (h); BPM11 (i); BPM12 (l); BPM13 (m); BPM14 (n); BP44 (o).

The composition data estimated by TGA and DSC are in excellent agreement with those calculated (Table 2).

In case of samples with high PTFE content, it is extremely difficult to obtain good SEM images because an excessive increase in the SEM acceleration voltage degrades the 


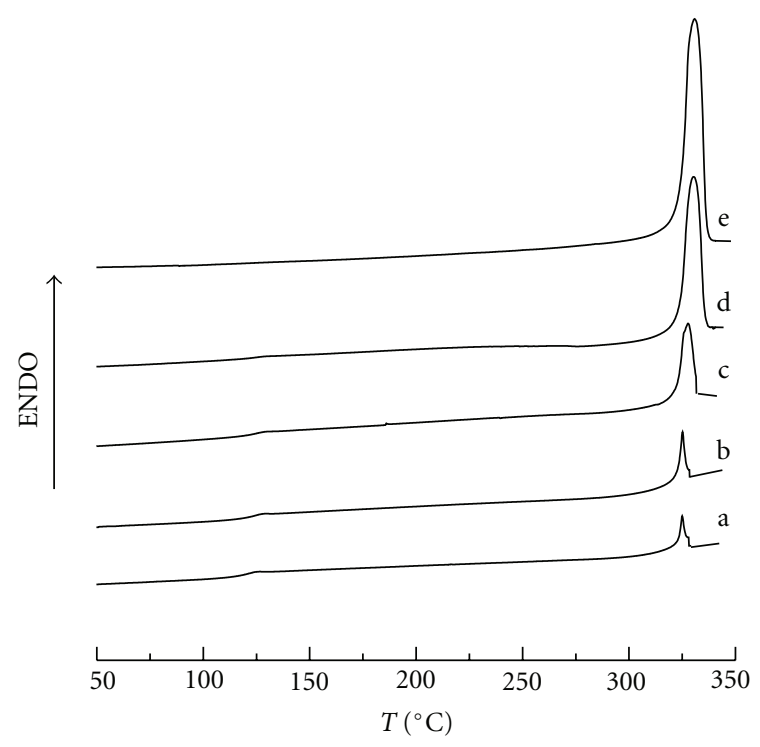

Figure 5: DSC second heating at $10^{\circ} \mathrm{C} / \mathrm{min}$ of samples: BPM4 (a), BPM5 (b), BPM7 (c), BPM11 (d), and BP44 (e).

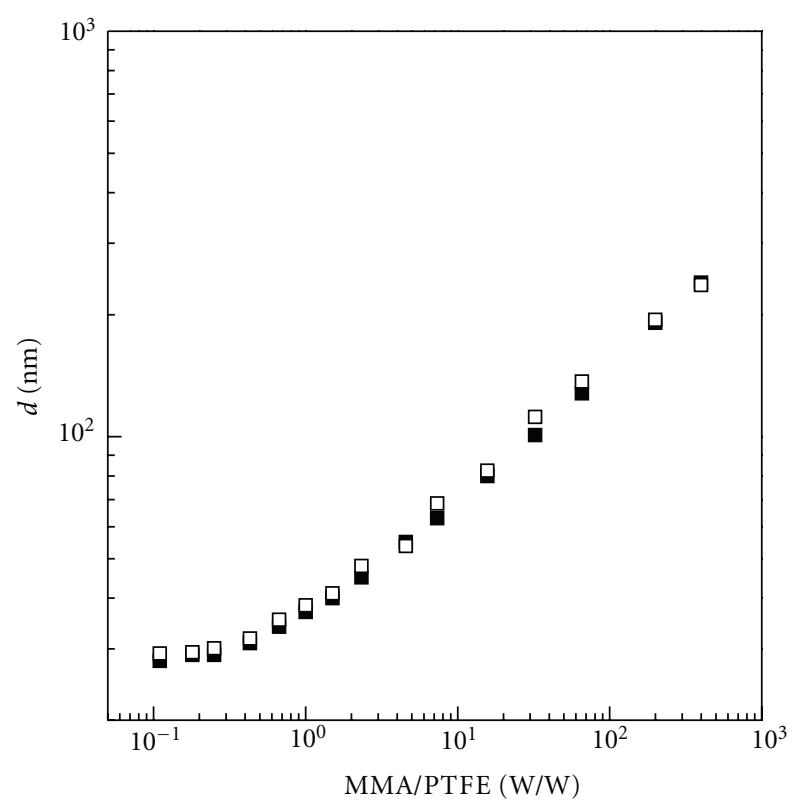

Figure 6: Trend of the particle diameter of the BPMn series as a function of the weight ratio between the initially added methyl methacrylate and PTFE. The full symbols refer to the calculated data, whereas the open symbols refer to the experimental results.

samples thus severely reducing the image quality. Consequently, to give a homogeneous evaluation of the size of the entire sample series, PCS data were collected (Table 2) and the relevant average diameters are reported in a double $\log$ scale in Figure 6 as a function of the weight ratio between the initial methyl methacrylate and PTFE. The full symbols in Figure 6 represent the expected final particle assuming no secondary nucleation or aggregation. Over the entire MMA/PTFE range, the particle size increases as the
MMA/PTFE ratio increases and agrees with the expected final particle size, indicating that secondary nucleation was prevented with all the monomer ending up as shell polymer. The systematic overestimation of the particle size with respect to the calculated size could derive from the inherent tendency of the PCS technique to overestimate the particle size with respect to the SEM data. To make a comparison, in case of sample BPM3 prepared with a ratio MMA/PTFE = 66 , the estimated size is $128 \mathrm{~nm}$ whereas the PCS and the SEM data are 136 and $132 \mathrm{~nm}$, respectively.

The overall picture of these data clearly indicates that a very precise control over the particle size can be exerted by properly adjusting the ratio between the monomer and the PTFE seed. The inhibition of the secondary nucleation is probably motivated by the large seed surface due to the very small size of the PTFE seeds. The wide surface area of the seed particles can capture all the unstable nuclei produced in the system even at very high MMA/PTFE ratios, thus avoiding the formation of stable secondary particles.

Figure 7 reports the SEM images of samples BPM1, BPM2, BPM4, and BPM6. The former three samples present average diameters of 236, 197, and $105 \mathrm{~nm}$ with uniformity ratios [16] $U$ of 1.01, 1.01, and 1.03, respectively, whereas for sample BPM6 an increase in the size distribution can be observed, although a reliable determination of the relevant $U$ value is not possible. The decrease in the $U$ value as the ratio MMA/PTFE increases indicates a self-sharpening propensity for these systems, as previously observed for other systems [17]. This behavior suggests the occurrence of a competitive growth mechanism [18] of latex particles in which small latex particles grow faster in size than larger ones, thus leading to narrow size distributions. It is interesting to note that this behavior was also observed [19] for the seeded dispersion polymerization of methyl methacrylate using PMMA submicron seeds. According to the above results, and considering that $U$ of about 1.05 is the upper limit to produce a good nanoparticle structuring [20], we can conclude that, using the above seeded polymerization technique, $2 \mathrm{D}$ and $3 \mathrm{D}$ colloidal crystals from particles in the $80-240 \mathrm{~nm}$ range can be prepared.

Compact nanocomposites were prepared by introducing the core-shell particles, as powder samples, into a rectangular mould. After an equilibration time at room temperature, the mould is heated to $160^{\circ} \mathrm{C}$, maintained at this temperature for 15 min under pressure, and then cooled to room temperature. No structural ordering was attempted and macroscopic samples in the form of rectangular sheets of $30 \times 5 \times$ $2 \mathrm{~mm}$ size were obtained. Figure 8 illustrates the effect of the nanoparticle size and the PTFE content on the corresponding lattices and nanocomposites appearance. Latexes containing relatively big particles appear milky, as can be seen for latex BPM4 (sample A), whereas as the particle size decreases the latexes become progressively more transparent. In contrast, the nanocomposites are transparent up to a very high PTFE content. The transparency is not totally lost also in case of sample BPM11 (sample D) that contains about $80 \%$ of PTFE.

This observation is a clear proof for an excellent dispersion of the PTFE particles within the polymer matrix. 


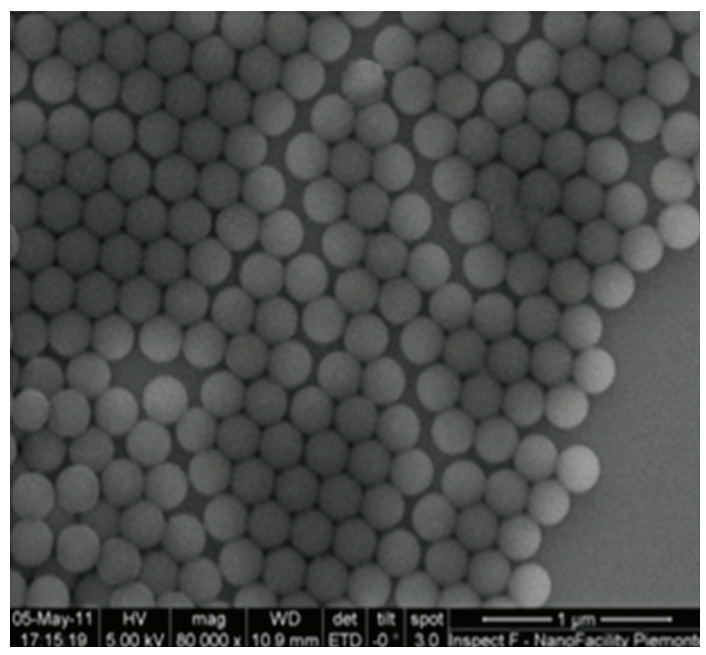

(a)

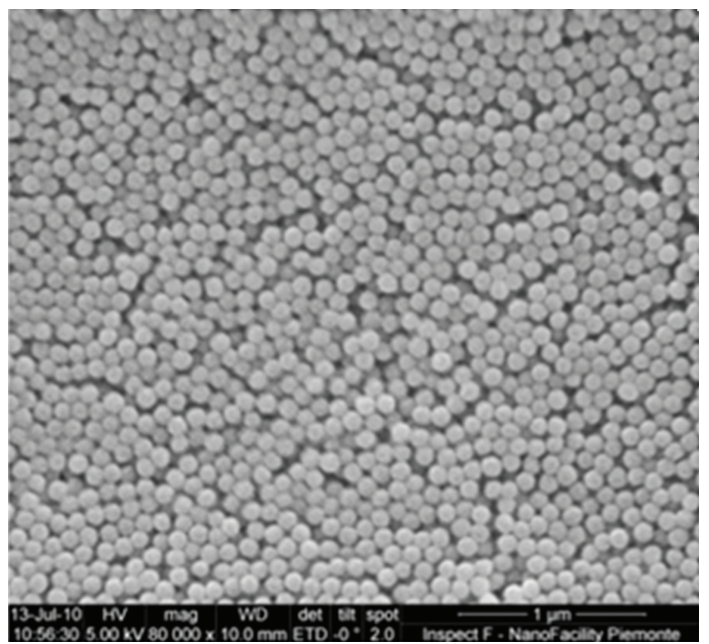

(c)

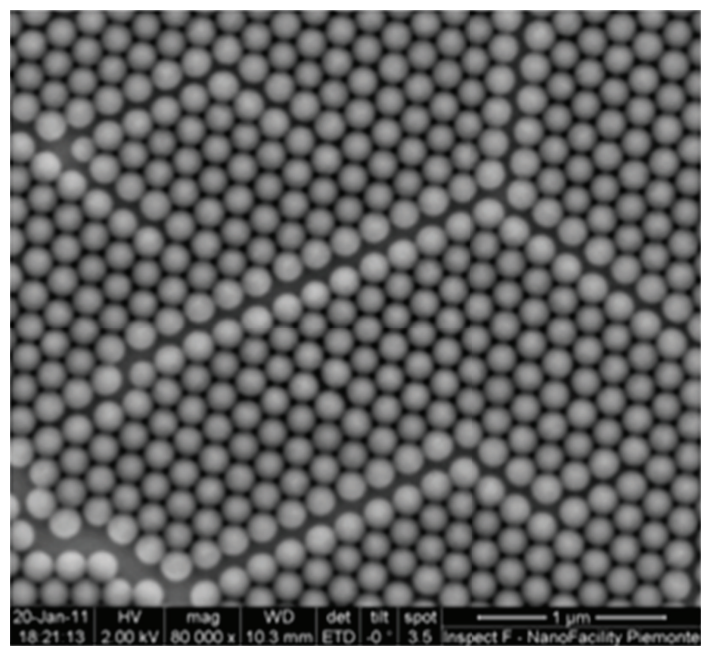

(b)

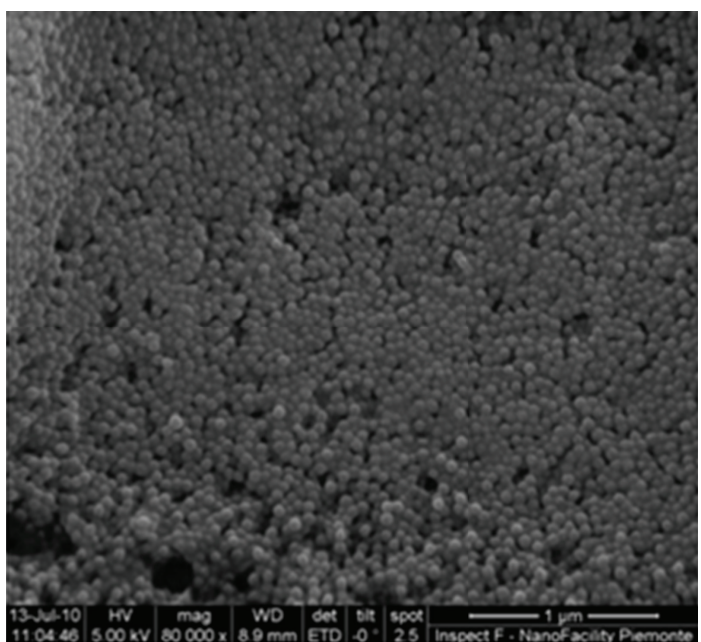

(d)

FIgURE 7: SEM micrographs of samples BPM1 (a), BPM2 (b), BPM4 (c), and BPM6 (d).

This result is also confirmed by the nanocomposite thermal behavior. In general, the melting of the PTFE occurs, with a heating rate of $10^{\circ} \mathrm{C} / \mathrm{min}$, at $325^{\circ} \mathrm{C}$ (Figure 5), whereas the minimum of the crystallization exotherm, with a cooling rate of $-10^{\circ} \mathrm{C} / \mathrm{min}$, is observed at $316^{\circ} \mathrm{C}$ (Figure 9(a)), in agreement with literature data [21]. The crystallization of the PTFE in the nanocomposites at $-20^{\circ} \mathrm{C} / \mathrm{min}$ is observed with minimum at $270^{\circ} \mathrm{C}$ (Figure $9(\mathrm{~b}-\mathrm{e})$ ). This peculiar crystallization behavior, recently described and thoroughly discussed [22] for a series of PTFE-PMMA core-shell nanoparticles, can be rationalized within the frame of the fractionated crystallization mechanism [23, 24]. Due to the compartmentalization of PTFE within the core-shell nanoparticles, the number of the dispersed PTFE particles is much greater than the number of heterogeneities that usually nucleate the polymer in bulk. In these conditions, only one crystallization component at very high undercooling is observed, deriving from the homogeneous nucleation mechanism $[25,26]$. In turn, the observation of the single low-temperature crystallization exotherm can be viewed as a proof for the occurrence of a perfect dispersion of the PTFE particles in the nanocomposite.

The dynamic mechanical behavior of the nanocomposites was also studied by DMTA using the three-point bending geometry in the linear viscoelasticity region at the frequency of $1 \mathrm{~Hz}$, between $40^{\circ} \mathrm{C}$ and the temperature at which the samples lost their dimensional stability, with a heating rate of $4^{\circ} \mathrm{C} / \mathrm{min}$. Figure 10 illustrates collectively the trends of the storage modulus $E^{\prime}$ and $\tan \delta$ as a function of temperature. The dynamic storage modulus $E^{\prime}$ decreases with increasing temperature with a drop at about $145^{\circ} \mathrm{C}$, corresponding to the glass transition of the PMMA component.

The glass transition temperature of the PMMA component is slightly affected by the PTFE amount and only a small increase in temperature, with a parallel broadening of the relaxation process in the high-temperature side of 


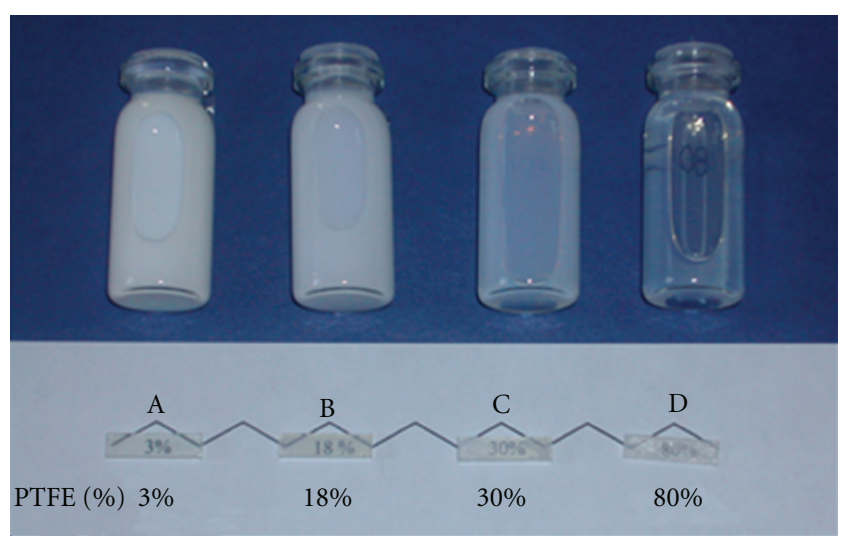

Figure 8: (A): BPM4 $(d=112 \mathrm{~nm}, 3 \%$ PTFE); (B): BPM7 $(d=$ $54 \mathrm{~nm}, 18 \%$ PTFE); (C): BPM8 (48 nm, 30\% PTFE); (D): BPM11 $(d=35 \mathrm{~nm}, 80 \%$ PTFE $)$.

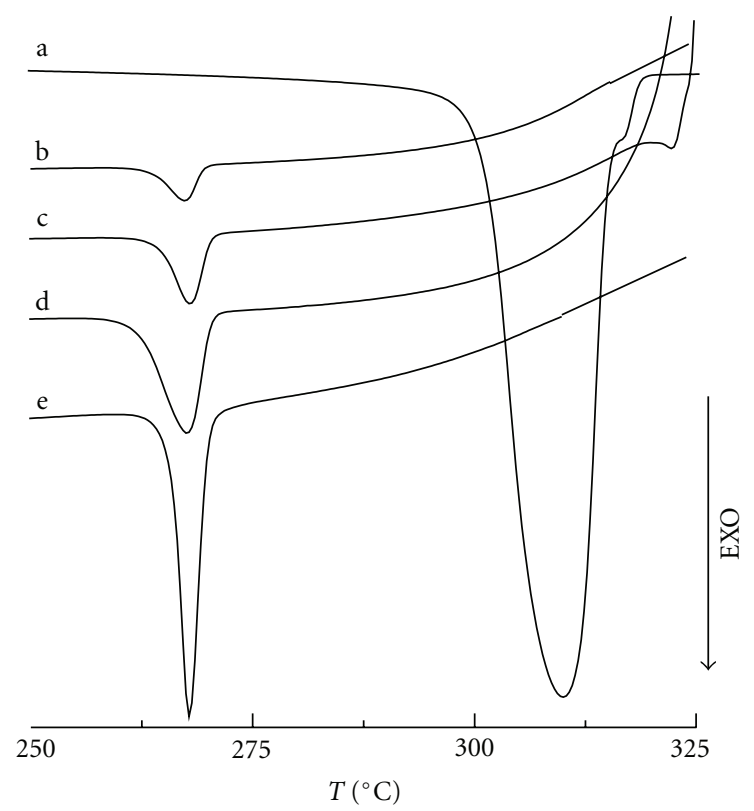

Figure 9: DSC cooling at $20^{\circ} \mathrm{C} / \mathrm{min}$ after fast heating to $330^{\circ} \mathrm{C}$ of samples BP44 (a), BPM4 (b), BPM5 (c), BPM6 (d), and BPM8 (e).

the transition is observed (Tables 2 and 3, Figure 10(B)). Figure 11 illustrates the trend of the storage modulus before and after the glass transition temperature. At a temperature lower that the PMMA glass transition, $E^{\prime}$ results about 2.5 3.5 $\mathrm{GPa}$, irrespective of the PTFE amount. In contrast, at temperatures higher than the PMMA glass transition, the storage modulus increases linearly as the PTFE amount increases. These effects are reported in many nanostructured material systems such as PMMA/silica [27] and PS/clay nanocomposites [28] and can be explained in terms of the classical mechanical reinforcement by the relatively rigid particles and restricted mobility in the polymer matrix [29].
TABle 3: Dynamic storage modulus $E^{\prime}$ in the glassy $\left(50^{\circ} \mathrm{C}\right)$ and rubbery $\left(200^{\circ} \mathrm{C}\right)$ state and glass transition temperatures from the maximum in the $\tan \delta$ peak.

\begin{tabular}{lccc}
\hline Sample & $\begin{array}{c}E^{\prime} \text { glassy state } 50^{\circ} \mathrm{C} \\
(\mathrm{GPa})\end{array}$ & $\begin{array}{c}E^{\prime} \text { rubbery state } 200^{\circ} \mathrm{C} \\
(\mathrm{MPa})\end{array}$ & $\begin{array}{c}T_{g} \\
\left({ }^{\circ} \mathrm{C}\right)\end{array}$ \\
\hline PMMA & 2.6 & 4.3 & 142 \\
BPM4 (3\%) & 2.8 & 6.9 & 146 \\
BPM5 (6\%) & 2.8 & 8.8 & 148 \\
BPM6 (12\%) & 3.0 & 9.0 & 148 \\
BPM7 (18\%) & 3.3 & 9.4 & 149 \\
BPM8 (30\%) & 3.4 & 12.6 & 148 \\
BPM9 (40\%) & 2.8 & 14.3 & 149 \\
BPM10 (50\%) & 2.9 & 15.9 & 149 \\
BPM11 (60\%) & 3.1 & 18.9 & 146 \\
\hline
\end{tabular}

\section{Conclusions}

This paper is addressed to the preparation of PTFEPMMA core-shell particles featuring controlled size and narrow size distribution over a wide compositional range, through an emulsifier-free seeded emulsion polymerization starting from a PTFE seed of 26 nanometers. Over the entire MMA/PTFE range, the particle size increases as the MMA/PTFE ratio increases and a very precise control over the particle size can be exerted by properly adjusting the ratio between the monomer and the PTFE seed. In addition, the particle size distribution self-sharpens as the ratio MMA/PTFE increases. Consequently, particles in the 80$240 \mathrm{~nm}$ range were prepared featuring uniformity indexes suited to build 2D and 3D colloidal crystals.

Compact nanocomposites can be prepared by annealing the core-shell particles at a temperature higher than the glass transition temperature of PMMA. Transparent nanocomposites are obtained up to a very high PTFE content thus indicating an excellent dispersion of the PTFE particles within the PMMA matrix which is also confirmed by the observation of fractionated crystallization effects of the PTFE particles. The dynamic mechanical behavior of various nanocomposites indicates that the glass transition of the PMMA component is slightly affected by the PTFE amount. The storage modulus, at temperatures lower than the PMMA glass transition is nearly constant in the various samples, whereas it increases linearly as the PTFE amount increases at temperatures higher that the PMMA glass transition as expected in case of a mechanical reinforcement by the relatively rigid particles and restricted mobility in the polymer matrix.

\section{Disclosure}

All the authors do not have any direct financial relation with the commercial identity mentioned in the paper that might lead to a conflict of interests. 


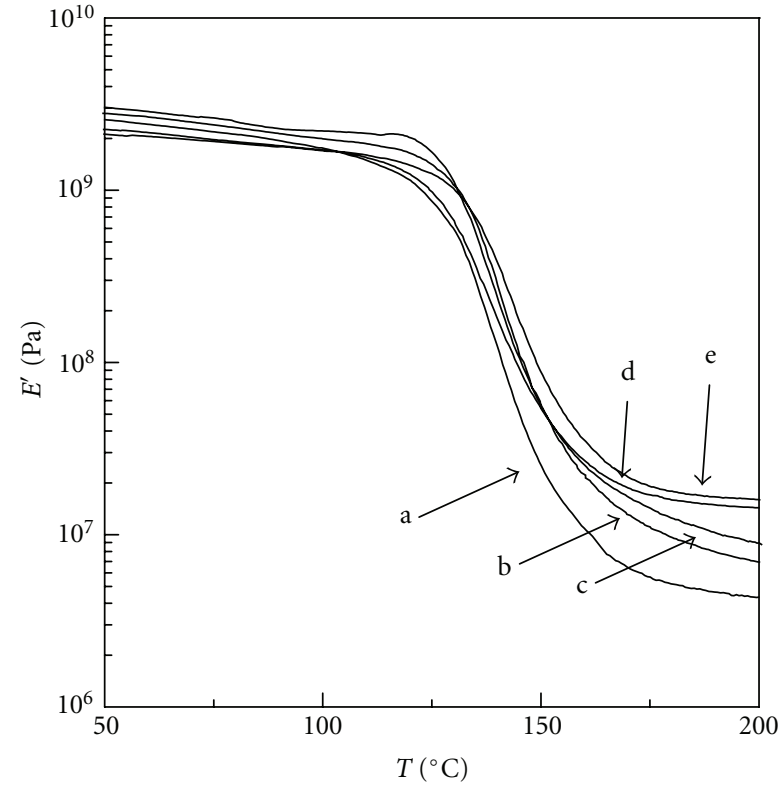

(A)

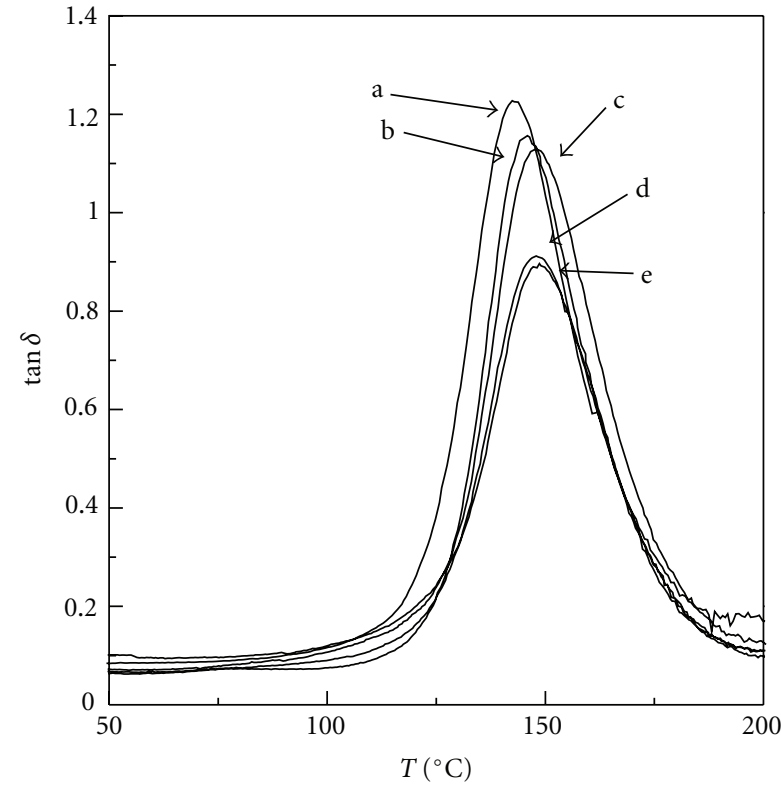

(B)

Figure 10: Trend of the storage modulus $E^{\prime}$ (A) and loss tangent $\tan \delta$ (B) for BPMn samples and pure PMMA sample as a function of temperature: PMMA (a), BPM4 (b), BPM6 (c), BPM9 (d), and BPM10 (e).

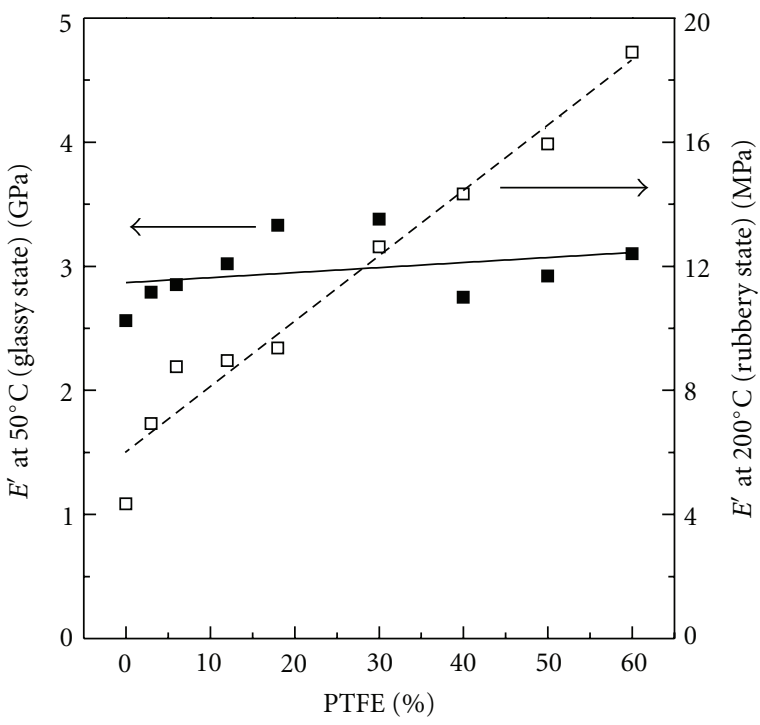

Figure 11: Trend of the storage modulus $E^{\prime}$ in glassy (full symbols, $\mathrm{GPa}$ ) and rubbery (open symbols, $\mathrm{MPa}$ ) states.

\section{Acknowledgment}

Part of this work has been performed at NanoFacility Piemonte, INRiM, a laboratory supported by Compagnia di San Paolo.

\section{References}

[1] E. P. Giannelis, "Polymer layered silicate nanocomposites," Advanced Materials, vol. 8, no. 1, pp. 29-35, 1996.
[2] P. M. Ajayan and J. M. Tour, "Materials science: nanotube composites," Nature, vol. 447, no. 7148, pp. 1066-1068, 2007.

[3] B. A. Rozenberg and R. Tenne, "Polymer-assisted fabrication of nanoparticles and nanocomposites," Progress in Polymer Science, vol. 33, no. 1, pp. 40-112, 2008.

[4] D. R. Paul and L. M. Robeson, "Polymer nanotechnology: nanocomposites," Polymer, vol. 49, no. 15, pp. 3187-3204, 2008.

[5] T. Wang and J. L. Keddie, "Design and fabrication of colloidal polymer nanocomposites," Advances in Colloid and Interface Science, vol. 147-148, no. 1, pp. 319-332, 2009.

[6] Y. Xia, B. Gates, Y. Yin, and Y. Lu, "Monodispersed colloidal spheres: old materials with new applications," Advanced Materials, vol. 12, no. 10, pp. 693-713, 2000.

[7] O. Kalinina and E. Kumacheva, "Polymeric nanocomposite material with a periodic structure," Chemistry of Materials, vol. 13, no. 1, pp. 35-38, 2001.

[8] U. Jeong, Y. Wang, M. Ibisate, and Y. Xia, "Some new developments in the synthesis, functionalization, and utilization of monodisperse colloidal spheres," Advanced Functional Materials, vol. 15, no. 12, pp. 1907-1921, 2005.

[9] J. C. Hulteen and R. P. Van Duyne, "Nanosphere lithography: a materials general fabrication process for periodic particle array surfaces," Journal of Vacuum Science and Technology A, vol. 13, no. 3, pp. 1553-1558, 1995.

[10] K. Inoue and K. Kazuo, "Photonic crystals physics, fabrication and applications," in Ohtaka editor Springer Series in Optical Sciences, vol. 94, Springer, Berlin, Germany, 2004.

[11] E. Giani, K. Sparnacci, M. Laus, G. Palamone, V. Kapeliouchko, and V. Arcella, "PTFE-polystyrene core-shell nanospheres and nanocomposites," Macromolecules, vol. 36, no. 12, pp. 4360-4367, 2003.

[12] K. Sparnacci, D. Antonioli, S. Deregibus et al., "PTFE-based core-soft shell nanospheres and soft matrix nanocomposites," Macromolecules, vol. 42, no. 10, pp. 3518-3524, 2009. 
[13] V. Kapeliouchko, G. Palamone, T. Poggio et al., "PMMA-based core-shell nanoparticles with various PTFE cores," Journal of Polymer Science A, vol. 47, no. 11, pp. 2928-2937, 2009.

[14] K. Sparnacci, D. Antonioli, S. Deregibus et al., "Twodimensional non-close-packed arrays of nanoparticles via core-shell nanospheres and reactive ion etching," Polymers for Advanced Technologies, vol. 23, no. 3, pp. 558-564, 2011.

[15] D. Antonioli, S. Deregibus, G. Panzarasa et al., "PTFE-PMMA core-shell nanoparticles as building blocks for self-assembled opals: synthesis, properties and optical response," Polymer International, vol. 61, no. 8, pp. 1294-1301, 2012.

[16] S. M. Heard, F. Grieser, C. G. Barraclough, and J. V. Sanders, "The characterization of ag sols by electron microscopy, optical absorption, and electrophoresis," Journal of Colloid And Interface Science, vol. 93, no. 2, pp. 545-555, 1983.

[17] K. Sparnacci, D. Antonioli, S. Deregibus et al., "Preparation, properties and self-assembly behavior of PTFE-based coreshell nanospheres," Journal of Nanomaterials, vol. 2012, Article ID 980541, 15 pages, 2012.

[18] C. S. Chern, Principles and Applications of Emulsion Polymerization, Wiley, New York, NY, USA, 2008.

[19] S. Jiang, E. D. Sudol, V. L. Dimonie, and M. S. El-Aasser, "Seeding as a means of controlling particle size in dispersion polymerization," Journal of Applied Polymer Science, vol. 108, no. 6, pp. 4096-4107, 2008.

[20] M. E. Woods, J. S. Dodge, I. M. Krieger, and P. E. Pierce, "Monodisperse lattices: emulsion polymerization with mixtures of anionic and nonionic surfactants," Journal of Paint Technology, vol. 40, no. 527, pp. 541-548, 1968.

[21] X. Q. Wang, D. R. Chen, J. C. Han, and S. Y. Du, "Crystallization behavior of polytetrafluoroethylene (PTFE)," Journal of Applied Polymer Science, vol. 83, no. 5, pp. 990-996, 2002.

[22] M. Laus, K. Sparnacci, D. Antonioli et al., "On the multiple crystallization behavior of PTFE in PMMA/PTFE nanocomposites from core-shell nanoparticles," Journal of Polymer Science B, vol. 48, no. 5, pp. 548-554, 2010.

[23] J. A. Koutsky, A. G. Walton, and E. Baer, "Nucleation of polymer droplets," Journal of Applied Physics, vol. 38, no. 4, pp. 1832-1839, 1967.

[24] R. Montenegro, M. Antonietti, Y. Mastai, and K. Landfester, "Crystallization in miniemulsion droplets," Journal of Physical Chemistry B, vol. 107, no. 21, pp. 5088-5094, 2003.

[25] G. Pompe, L. Häußler, P. Pötschke et al., "Reactive polytetrafluoroethylene/polyamide compounds. I. Characterization of the compound morphology with respect to the functionality of the polytetrafluoroethylene component by microscopic and differential scanning calorimetry studies," Journal of Applied Polymer Science, vol. 98, no. 3, pp. 1308-1316, 2005.

[26] L. Häußler, G. Pompe, D. Lehmann, and U. Lappan, "Fractionated crystallization in blends of functionalized poly(tetrafluoroethylene) and polyamide," Macromolecular Symposia, vol. 164, no. 1, pp. 411-420, 2001.

[27] Y. H. Hu, C. Y. Chen, and C. C. Wang, "Viscoelastic properties and thermal degradation kinetics of silica/PMMA nanocomposites," Polymer Degradation and Stability, vol. 84, no. 3, pp. 545-553, 2004.

[28] R. Ruggerone, C. J. G. Plummer, N. N. Herrera, E. BourgeatLami, and J. A. E. Månson, "Highly filled polystyrene-laponite nanocomposites prepared by emulsion polymerization," European Polymer Journal, vol. 45, no. 3, pp. 621-629, 2009.

[29] L. E. Nielsen and R. W. Landel, Mechanical Properties of Polymers and Composites, Marcel Dekker, New York, NY, USA, 1994. 

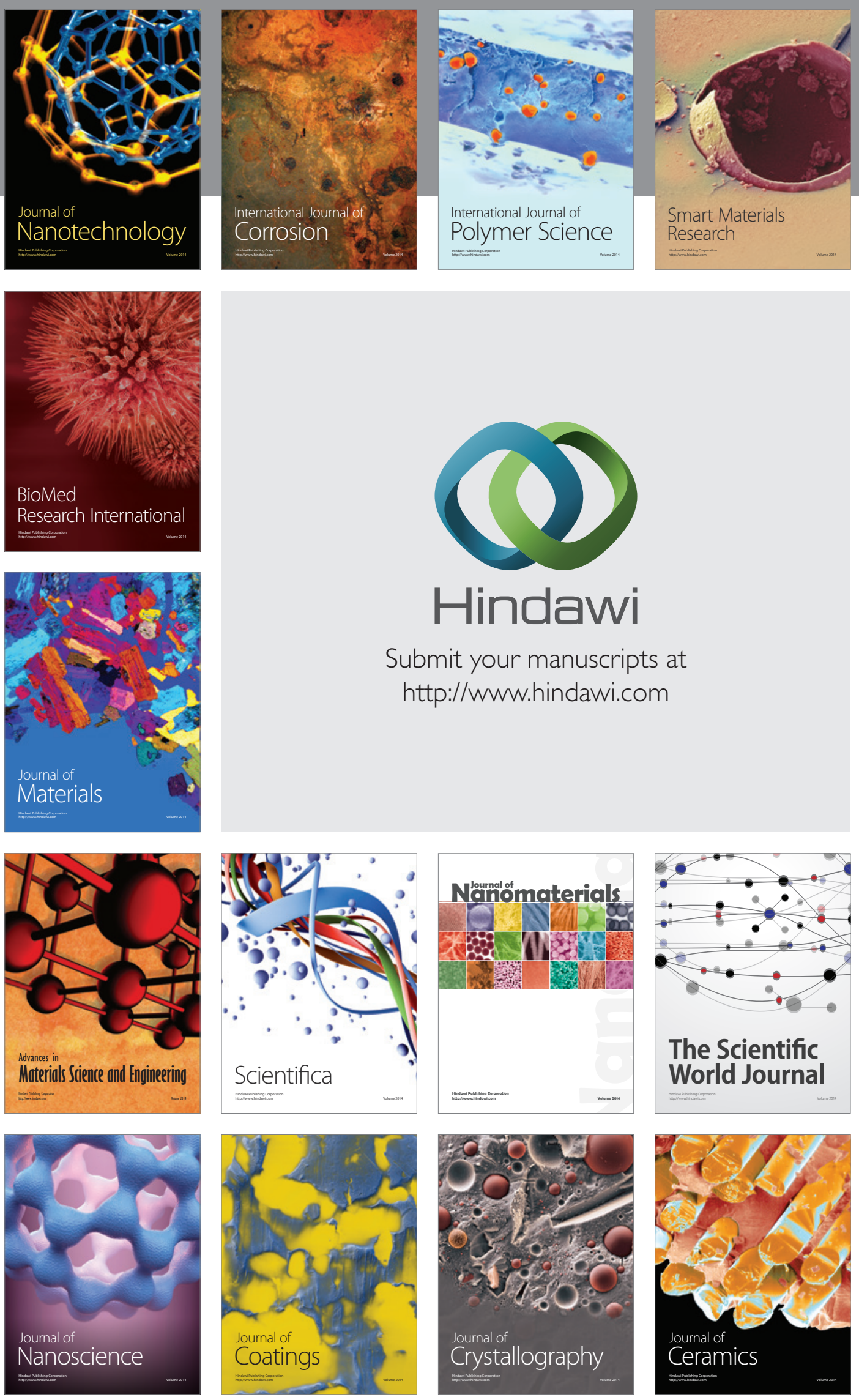

The Scientific World Journal

Submit your manuscripts at

http://www.hindawi.com

\section{World Journal}

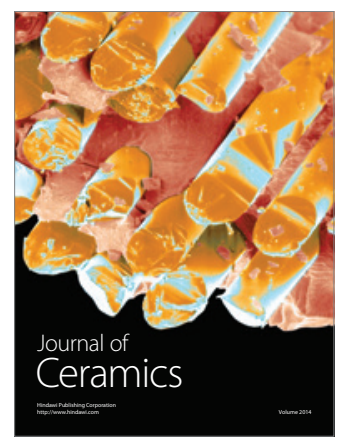

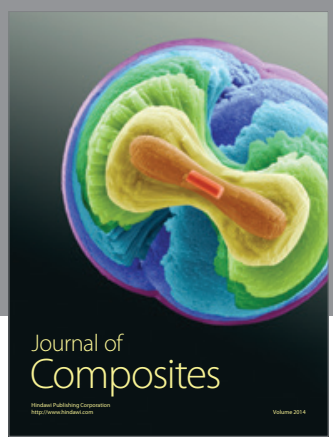
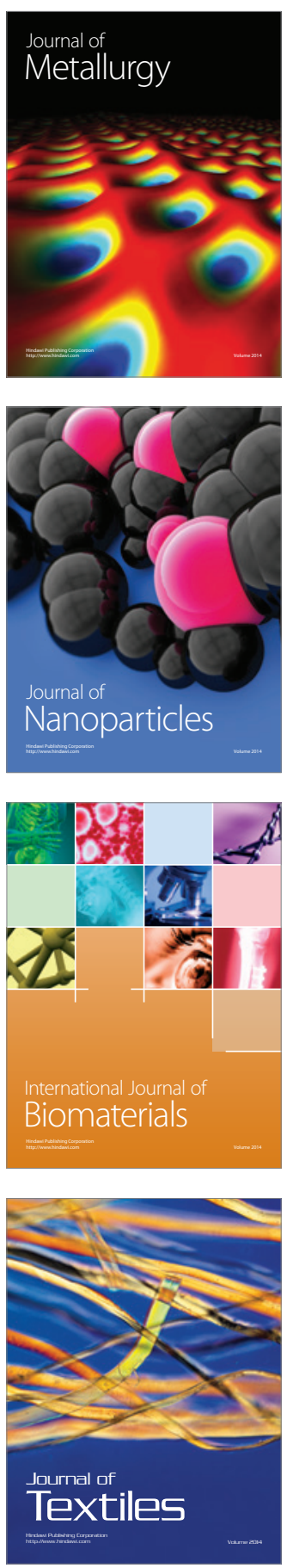